\title{
Experiences of Adults Using Feedback-Based Technology to Improve Physical Function in Rehabilitation: Study Protocol for a Qualitative Systematic Review
}

\author{
Caitlin Hamilton', Annie McCluskey', Meryl Lovarini' ${ }^{1}$, \\ Tarcisio Folly Campos ${ }^{2}$, Leanne Hassett ${ }^{1,2}$ \\ ${ }^{1}$ Faculty of Health Sciences, The University of Sydney, Sydney, Australia \\ ${ }^{2}$ The George Institute for Global Health, Sydney Medical School, The University of Sydney, Sydney, Australia \\ Email: cham9802@uni.sydney.edu.au
}

Received 3 June 2016; accepted 30 July 2016; published 2 August 2016

Copyright (C) 2016 by authors and Scientific Research Publishing Inc.

This work is licensed under the Creative Commons Attribution International License (CC BY).

http://creativecommons.org/licenses/by/4.0/

(c) (i) 0pen Access

\begin{abstract}
Question: What are the experiences of adults using feedback-based technology to improve their physical function during rehabilitation? Design: A systematic review with meta-synthesis of qualitative studies is proposed. Participants: Adults (aged $\geq 15$ years) participating in neurological or aged care rehabilitation. Intervention: Feedback-based technology (technology providing feedback to the user about task performance or dose of practice) used as an intervention to improve physical function or physical activity levels. Outcomes: Experiences of using feedback-based technology (learning, accepting, adopting, practicing and progressing), as well as barriers and facilitators to use. Methods: Meta-synthesis of qualitative studies involving the following steps: 1) a systematic search of 10 electronic databases and grey literature, and hand-searching of reference lists of included studies, 2) screening of search results and abstracts for study selection, 3) data extraction, 4) quality appraisal of included studies using the Critical Appraisal Skills Programme (CASP) checklist, and 5) qualitative synthesis to identify common themes and concepts across studies. The Enhancing Transparency in Reporting the Synthesis of Qualitative Research (ENTREQ) statement will be used to report study methods and results. Discussion: This meta-synthesis will provide new knowledge about how feedback-based technologies are currently being used in rehabilitation, and describe users' experiences. Review registration: PROSPERO International prospective register of systematic reviews Registration No. CRD42015017119.
\end{abstract}

\section{Keywords}

Rehabilitation, Feedback, Technology, Qualitative, Physical 


\section{Introduction}

Reduced physical function resulting from impairments associated with conditions such as stroke, brain injury, amputations and hip fracture is common. Engagement in therapeutic activities during rehabilitation can improve physical function if practice is task specific, repetitive and intensive [1] [2]. Extrinsic augmented feedback about performance can improve physical function by enhancing motor learning [3] [4]. Many technologies provide extrinsic feedback to users about their performance and practice completed. This feedback may facilitate engagement and increase the dose of repetitive exercise completed during rehabilitation. Feedback-based technologies are becoming more affordable and accessible, making them viable for use in rehabilitation settings to train physical function and promote physical activity. These technologies include virtual reality systems which provide users with feedback about movement in simulated or real life environments, and games (e.g. CAREN, Motekforce Link, Amsterdam, The Netherlands; Nintendo Wii, Nintendo, Kyoto, Japan), wearable devices which provide feedback about movement (e.g. step count from a Fitbit activity monitor, Fitbit Inc., San Francisco, California, USA), and biofeedback devices which provide feedback about unconscious physiological processes (e.g. centre of pressure/mass from a force platform).

The use of feedback-based technologies to improve physical function has been evaluated in clinical trials and the results synthesised in systematic reviews [5]-[8]. Most studies have investigated the use of virtual reality technologies (both commercially available and customised systems), and most focus on the stroke population. Key findings from these systematic reviews included improvements in walking speed, balance, mobility [5] and upper limb function [6] [8] when virtual reality was compared with the same dose of conventional therapy. Similarly, greater improvements in lower limb activities were reported when biofeedback was used during practice by people following stroke [7]. However, there is still uncertainty about the effectiveness of interventions that use feedback-based technologies due to small study samples and poor reporting of study methods. Larger clinical trials are now being conducted in this area, as well as research led by technology developers to design and evaluate new technologies suitable for use in rehabilitation. As researchers conduct these larger scale clinical trials, and developers of technology start to work more with the end users of these systems (i.e. therapists and patients), the preferences and experiences of patients have been explored.

Patient preferences and experiences of using feedback-based technologies in rehabilitation have been explored using quantitative and qualitative methods, but with mixed results. For example, Chen and colleagues [9] found that patient participants were positive about using virtual reality gaming technologies in rehabilitation, whereas Laver and colleagues [10] found that patients preferred conventional face-to-face individual therapy rather than a program using virtual reality systems. Research on patient preferences and experiences has been synthesised in a small number of reviews [8] [11]. The main finding to emerge from these reviews was that the use of technology enhanced patients enjoyment and engagement in therapy. Furthermore, experiencing a sense of achievement whilst continuously being challenged to improve one's ability seems to be key to patient enjoyment. Limitations of these reviews include the small number of studies, focus on the use of virtual reality in a neurological population only, and studies published only up to January 2013. The types of feedback-based technologies available for use in rehabilitation settings is growing rapidly, and many qualitative studies exploring patient preferences and experiences are being published. Therefore, an updated review is warranted, including a wider range of feedback-based technologies and non-neurological populations.

A systematic review of qualitative studies exploring patient experiences of using feedback-based technology in rehabilitation is proposed. The primary aim of this review will be to identify and synthesise the findings of qualitative studies that explore the experience of adults who have used feedback-based technologies in physical rehabilitation. A secondary aim will be to build a new theory or theories to better understand and explain how technology can enhance skill acquisition and motivate patients to achieve the high dose of exercise associated with improved physical outcomes. This review also has the potential to assist with user-focused technology development. The review will explore characteristics of studies to identify any conditions and/or criteria that influence patient experiences, for example, the setting, diagnosis, age or types of technologies. In addition, the review aims to identify any gaps in knowledge and inform the direction of future qualitative research. The outcomes in this review will be restricted to physical function. A parallel review is being conducted by the authors on therapists' experiences of using feedback-based technology in rehabilitation (Prospero Registration number: CRD42015020271). 


\section{Methods}

\subsection{Design}

The study design is a meta-synthesis of qualitative studies, comprising the following steps: 1) a systematic search of 10 electronic databases and grey literature, and hand searching of reference lists of included studies, 2) screening of search results and study selection, 3) data extraction, 4) quality appraisal of the included studies using the Critical Appraisal Skills Programme (CASP) checklist [12], and 5) qualitative synthesis to identify common themes and concepts across studies. The review protocol was registered on the International Prospective Register of Systematic Reviews (PROSPERO) on 27 February 2015 and last updated on 7 December 2015 [Registration number: CRD42015017119]. Any protocol amendments will be tracked and include the date of each amendment, description of the change and the rationale. The Enhancing Transparency in Reporting the Synthesis of Qualitative Research (ENTREQ) statement [13] will be used for reporting study methods and results.

\subsection{Eligibility Criteria}

The focus of the review is on the experience of adults who have used feedback-based technology to improve their physical function during rehabilitation. Studies will be judged for relevance according to the eligibility criteria outlined in Table 1. Studies will be excluded for the following reasons: 1) conference proceedings, 2) technology is used for other rehabilitative purposes, such as the provision of information/education, assessment, maintenance of records, as a medium to provide therapy (e.g. tele-health) or as an assistive device, 3) technology is used for improving other functions such as cognition, vision and pain and 4) report is on the experiences of caregivers, family members or therapists rather than the experiences of the adult/s participating in rehabilitation.

\subsection{Study Identification}

\subsubsection{Information Sources}

Ten electronic databases will be searched including: MEDLINE, CINAHL, Web of Science Core Collection, AMED, PsycINFO, EMBASE, OTseeker, PEDro, COMPENDEX and IEEEXplore. Grey literature sources will include theses and dissertations and will be searched using the following electronic sources: ProQuest Dissertation \& Theses Full Text, British Library Electronic Digital Thesis Online Service (EThOS), and DART-Europe E-theses Portal. Studies will also be identified by searching the reference lists of included studies and contacting the first author of each identified study to ask if they are aware of any other published studies.

\subsubsection{Search Strategy}

A search strategy has been developed initially for Medline (Table 2) and will be adapted for each database in

Table 1. Eligibility criteria.

\begin{tabular}{|c|c|}
\hline Selection criteria & Inclusion criteria \\
\hline Study designs & $\begin{array}{l}\text { Peer reviewed and provides qualitative data obtained using empirical qualitative research approaches } \\
\text { Qualitative research approaches may include but not be limited to grounded theory, } \\
\text { ethnography, phenomenology and action participatory research. Studies using mixed methods } \\
\text { with a qualitative component will be included if it reports qualitative findings that are not numerical in nature }\end{array}$ \\
\hline Participants & Adults (aged 15 years and over) participating in aged care or neurological rehabilitation \\
\hline Intervention & $\begin{array}{l}\text { Technology used for rehabilitative purposes, for example as a therapy intervention or treatment } \\
\text { modality to improve physical function such as motor function, mobility or physical activity levels. } \\
\text { Type of technology used provides the user with feedback about task performance or dose of practice }\end{array}$ \\
\hline Outcomes & $\begin{array}{l}\text { Explores the experience of adults using technology during their rehabilitation, } \\
\text { for example learning, practising, progressing, accepting and adopting, as well as barriers and facilitators to use }\end{array}$ \\
\hline Setting & $\begin{array}{l}\text { Aged-care or neurological rehabilitation contexts for conditions such as, falls, amputations, stroke, } \\
\text { brain injury, spinal cord injury and other neurological conditions, such as Parkinson disease and } \\
\text { Multiple sclerosis; hospital (acute, sub-acute, rehabilitation), community (home, independent living units, } \\
\text { retirement villages) or residential aged care facility (hostels and nursing homes) settings }\end{array}$ \\
\hline Language & Published in English or can be translated into English within the resources available for the review \\
\hline
\end{tabular}


Table 2. Search strategy for medline.

\begin{tabular}{|c|c|}
\hline 1 & exp accidental falls/or exp amputees/ \\
\hline 2 & (“hip replacement*” or “knee replacement*” or “joint replacement*”).tw. \\
\hline 3 & (“older adult*” or “older person*” or "older people*” or elder* or senior* or geriatric*).tw. \\
\hline 4 & exp “aged, 80 and over”/or exp aged/or exp aging/or exp geriatrics/ \\
\hline 5 & (fall* or amput*).tw. \\
\hline 6 & $\begin{array}{l}\text { exp brain injuries/or exp stroke/or exp Parkinson Disease/or exp nervous } \\
\text { system diseases/or exp Multiple Sclerosis/or exp spinal cord injuries/ }\end{array}$ \\
\hline 7 & $\begin{array}{l}\text { (“brain injur*” or stroke or “cerebrovascular accident” or “Parkinson Disease” } \\
\text { or “multiple sclerosis” or “spinal cord injur*” or "guillain barre syndrome”).tw. }\end{array}$ \\
\hline 8 & $\begin{array}{l}\text { ("neurological disorder*” or "neurological disease*” or "neurological impairment*" or "neurologic disorder*” } \\
\text { or "neurologic disease*” or "nervous system disease*” or "nervous system disorder*”).tw }\end{array}$ \\
\hline 9 & 1 or 2 or 3 or 4 or 5 or 6 or 7 or 8 \\
\hline 10 & (“activity monitor*” or “activity tracker*” or pedometer* or acceleromet* or Fitbit or Garmin).tw. \\
\hline 11 & exp Video Games/or (videogam* or “video-gam*”).tw. \\
\hline 12 & (“computer gam*” or exergam* or “exer?gam*”).tw. \\
\hline 13 & (“virtual reality” or “virtual environment*”).tw. \\
\hline 14 & (biofeedback or “feedback device*” or "pressure sensor*”).tw. \\
\hline 15 & exp self-help device/or “self-help device*”.tw. \\
\hline 16 & (“assistive device*” or “assistive technology”).tw. \\
\hline 17 & exp robotics/or robotic*.tw. \\
\hline 18 & exp Computers, Handheld/or exp Software/or exp Cell Phone/ \\
\hline 19 & (“cellular phone*” or “cell phone*” or “mobile phone*” or smartphone* or iphone*).tw. \\
\hline 20 & ("mobile device*” or "handheld device*” or "handheld computer*” or "tablet computer*” or ipad*).tw. \\
\hline 21 & exp mobile applications/or (“mobile app*” or “tablet app*” or “phone app*”).tw. \\
\hline 22 & (Nintendo or Wii* or Kinect or Xbox or Playstation or Humac).tw. \\
\hline 23 & exp Monitoring, Ambulatory/or “ambulatory monitor*”.tw. \\
\hline 24 & (app or apps).tw. \\
\hline 25 & 10 or 11 or 12 or 13 or 14 or 15 or 16 or 17 or 18 or 19 or 20 or 21 or 22 or 23 or 24 \\
\hline 26 & exp motor activity/or exp exercise/or exp exercise tolerance/or exp physical endurance/or exp physical fitness/ \\
\hline 27 & (“physical activity” or “exercise” or “physical endurance” or “physical fitness”).tw. \\
\hline 28 & $\begin{array}{l}\text { (“balance” or “motor skills” or "coordination” or “motor function” or “muscle strength” } \\
\text { or "range of motion” or "range of movement”).tw. }\end{array}$ \\
\hline 29 & exp postural balance/or exp motor skills/or exp muscle strength/or exp “range of motion, articular"/ \\
\hline 30 & (“bed mobility” or sit* or "sit to stand” or stand* or transfer*).tw. \\
\hline 31 & (“functional reach” or grasp* or reach*).tw. \\
\hline 32 & exp walking/or exp gait/or exp hemiplegia/ \\
\hline 33 & (mobili* or walk* or gait or “stair climb*” or “stair walk*” or hemiplegi*).tw. \\
\hline 34 & (“motor learning” or “motor re?learning” or “motor training” or “task specific”).tw. \\
\hline 35 & exp Physical Therapy Modalities/or exp Occupational Therapy/ \\
\hline 36 & exp exercise movement techniques/or exp exercise therapy/ \\
\hline 37 & exp Recreation Therapy/or exp Therapy, Computer-Assisted/ \\
\hline 38 & exp Rehabilitation/ \\
\hline 39 & $\begin{array}{l}\text { (“physical therap*” or physiotherap* or “occupational therap*” or “exercise therap*” } \\
\text { or "exercise physiolog*” or "recreation therap*” or "computer assisted therap*” or rehabilit*).tw. }\end{array}$ \\
\hline 40 & 26 or 27 or 28 or 29 or 30 or 31 or 32 or 33 or 34 or 35 or 36 or 37 or 38 or 39 \\
\hline 41 & (experience* or perception* or perspective* or attitude* or preference* or belief* or feeling*).tw. \\
\hline 42 & (feasibility or barrier* or facilitat* or usability or suitability or learn* or practis*).tw. \\
\hline 43 & (accept* or adopt* or implement* or adher* or motivat* or engage* or satisfaction).tw. \\
\hline 44 & 41 or 42 or 43 \\
\hline 45 & 9 and 25 and 40 and 44 \\
\hline 46 & limit 45 to humans \\
\hline
\end{tabular}


consultation with a health sciences librarian. No language or publication date restrictions will be imposed other than those inherent within the design of each electronic database searched. Search terms will first be grouped according to whether they relate to the "population", "intervention" and "outcomes of interest", and then combined with "AND" (except when restricted by database design). To select appropriate search terms for each concept, previous studies on the topic will be reviewed. Pilot searches will be conducted to determine suitable search combinations. Search terms will include a mixture of database controlled keywords, including MESH headings and non-controlled keywords.

"Population" search terms will include people participating as patients in aged-care or neurological rehabilitation as inpatients or non-inpatients. "Intervention" search terms will be separated into three concepts: "technology", "physical therapies and modalities" and "physical rehabilitation interventions". The three concepts will be combined with Boolean operators as follows: "technology" AND ("physical therapies and modalities" OR "physical rehabilitation interventions").

The "technology" search terms will include a range of feedback-based technologies that patients may use to improve their physical function, including video and computer-based exercises and games, activity monitors, computer or phone devices and applications and biofeedback devices. "Physical therapies and modalities" search terms will include the main physical therapies and modalities that are used in rehabilitation, for example in physiotherapy, occupational therapy and exercise therapy. "Physical rehabilitation interventions" search terms will include the purpose of using technologies in rehabilitation, and use terms that are the focus of physical rehabilitation interventions such as exercise, physical activity, balance, strength, motor skills, walking, standing and reaching. The "outcomes of interest" search terms will include those used to describe or report on the experiences of using technology, for example experiences, perceptions, attitudes, beliefs, acceptance and adoption, barriers and facilitators.

\subsection{Selection of Studies}

Libraries will be created for search results retrieved from each database using Endnote, a reference management software package. Each library will be combined to form one master library in Endnote. Duplicate studies will be removed in Endnote as the libraries are combined. The selection of studies for the review will follow a three-stage process of initial exclusion, screening and final selection.

In Stage 1,one reviewer (CH) will undertake the following searches in Endnote: 1) Conference proceedings will be searched for using the terms conference and congress in the reference type and title, and then excluded, 2) Animal studies will be searched for using the match word function and searching the terms rat/s, mice, rabbit/s by title and abstract and then excluded, 3) Qualitative studies will be searched for by title and abstract, using truncation where appropriate using the terms qualitative, interview, focus group, thematic, grounded theory, ethnography, phenomenology, action research, mixed methods, questionnaire, survey, narrative, participant observation, observation, fieldwork, key informant, constant comparison, content analysis, discourse analysis, conversation analysis, interpretive, lived experience, hermeneutic and life world, and records not identified as qualitative then excluded. Terms will not be matched to facilitate a broader search for studies containing qualitative research.

In Stage 2, the title and abstract of studies identified during Stage 1 will be screened in Endnote independently by two reviewers (CH and TF) to identify potentially eligible studies. Screening outcomes will be compared and discrepancies resolved through discussion with a third reviewer (LH) to reach a consensus. In Stage 3, the full text of each study that potentially meets the inclusion criteria will be obtained and independently assessed for eligibility by at least two reviewers (CH and LH or TF). Any disagreements will be resolved through discussion. However, if consensus is not reached, the eligibility of the study will be decided following discussion with other team members (AM and ML). Study authors will be contacted to provide further information if the full text does not provide the information necessary to determine eligibility for the review.

\subsection{Risk of Bias (Quality) Assessment}

Studies included in the review will be appraised for methodological quality and trustworthiness to determine their significance to the review question. Studies will be assessed using The Critical Appraisal Skills Programme toolkit for qualitative studies (CASP) [12]. Items on the checklist will be independently assessed for their presence in each of the studies by two reviewers (CH and ML). Any discrepancies in appraisal will be resolved through discussion between review team members. 


\subsection{Data Extraction}

Data relevant to the aims of this review only will be extracted. For example, data reported in studies that relate to effectiveness of an intervention will not be extracted. Data extraction will be undertaken independently by two reviewers ( $\mathrm{CH}$ and $\mathrm{ML}$ ). Extracted data will be compared and discrepancies resolved through discussion with other team members (AM and LH).

A template will be used to extract variables of relevance. We will extract general information (year of publication, country, type of publication, type of study), participant characteristics (number, average age, gender, clinical diagnosis, eligibility criteria, time since injury/diagnosis, symptom/injury severity, ambulatory status, experience using technology prior to use in rehabilitation), details about the intervention (name of feedbackbased technology, description of the technology and context of use, physical outcomes of interest, co-intervention (if applicable) and methods of the study (aim/s of the study, methodological approach, sampling method, data collection methods, data analysis). A pilot trial of data extraction will be undertaken using a template. Study authors will be contacted to provide any missing or additional data required.

Text data will be extracted about the experiences of using feedback-based technology, that is, experiences of learning, practicing, progressing, accepting and adopting, as well as barriers and facilitators to use. Relevant data including study participant quotes and the author's interpretations provided in the findings, results, discussion or conclusion sections identified in the primary studies will be extracted. Text will be imported into NVivo10, a computer assisted qualitative data analysis software package, to assist data synthesis.

\subsection{Data Synthesis}

Data synthesis will take an inductive approach and be undertaken by at least two reviewers (CH and AM or ML). Data will be coded for content, then categorised into more focused themes based on our review question to identify patient experiences. Similarities and differences in the study findings will be compared, and links/relationships with the themes evident in patient experiences made in order to integrate the data and develop new insights. The heterogeneity of included studies based on theoretical or contextual differences will also be considered during this process.

Subgroup analysis will be undertaken if studies are sufficiently homogeneous. Sub-group analyses may explore differences in terms of clinical diagnosis (neurological compared to non-neurological), participant characteristics (age, gender), type of technology (commercially available virtual reality systems, customised virtual reality systems, wearable devices, biofeedback devices) and the context of technology use (hospital-based, homebased, community-based rehabilitation).

\section{Discussion}

The use of technologies to promote exercise during rehabilitation is important in the current healthcare climate and for the future, where there is an increasing demand for services using limited resources. Bridging the gap between evidence of benefit and practice is needed for successful implementation of technologies. Understanding patient preferences and experiences is critical to successful implementation and to provide consumer-driven health care. This study will provide knowledge and an increased understanding of how feedback-based technologies can best be used in rehabilitation to promote exercise and meet the needs of patients.

\section{Acknowledgements}

We would like to thank Elaine Tam, Academic Liaison Librarian at The University of Sydney for her assistance in the development of the search strategy.

\section{Funding}

CH is supported by a PhD scholarship from an Australian National Health and Medical Research Council Project Grant (APP1063751).

\section{References}

[1] Veerbeek, J.M., van Wegen, E., van Peppen, R., van der Wees, P.J., Hendriks, E., Rietberg, M. and Kwakkel, G. (2014) 
What Is the Evidence for Physical Therapy Poststroke? A Systematic Review and Meta-Analysis. PLoS ONE, 9, e87987. http://dx.doi.org/10.1371/journal.pone.0087987

[2] Olivetti, L., Schurr, K., Sherrington, C., Wallbank, G., Pamphlett, P., Kwan, M.M.-S. and Herbert, R.D. (2007) A Novel Weight-Bearing Strengthening Program during Rehabilitaiton of Older People Is Feasible and Improves Standing up More Than a Non-Weight-Bearing Strengthening Program: A Randomised Trial. Australian Journal of Physiotherapy, 53, 147-153. http://dx.doi.org/10.1016/S0004-9514(07)70021-1

[3] van Vliet, P.M. and Wulf, G. (2006) Extrinsic Feedback for Motor LEARNING after stroke: What Is the Evidence? Disability and Rehabilitation, 28, 831-840. http://dx.doi.org/10.1080/09638280500534937

[4] Boyd, L.A. and Winstein, C.J. (2001) Implicit Motor-Sequence Learning in Humans Following Unilateral Stroke: The Impact of Practice and Explicit Knowledge. Neuroscience Letters, 298, 65-69. http://dx.doi.org/10.1016/S0304-3940(00)01734-1

[5] Corbetta, D., Imeri, F. and Gatti, R. (2015) Rehabilitation that Incorporates Virtual Reality Is More Effective Than Standard Rehabilitation for Improving Walking Speed, Balance and Mobility after Stroke: A Systematic Review. Journal of Physiotherapy, 61, 117-124. http://dx.doi.org/10.1016/j.jphys.2015.05.017

[6] Laver, K.E., George, S., Thomas, S., Deutsch, J.E. and Crotty, M. (2015) Virtual Reality for Stroke Rehabilitation (Review). Cochrane Database of Systematic Reviews, No. 2. http://dx.doi.org/10.1002/14651858.cd008349.pub3

[7] Stanton, R., Ada, L., Dean, C. and Preston, E. (2011) Biofeedback Improves Activities of the Lower Limb after Stroke: A Systematic Review. Journal of Physiotherapy, 57, 145-155. http://dx.doi.org/10.1016/S1836-9553(11)70035-2

[8] Thomson, K., Pollock, A., Bugge, C. and Brady, M. (2014) Commercial Gaming Devices for Stroke upper Limb Rehabilitation: A Systematic Review. International Journal of Stroke, 9, 479-88. http://dx.doi.org/10.1111/ijs.12263

[9] Chen, M.-H., Huang, L.-L., Lee, C.-F., Hsieh, C.-L., Lin, Y.-C., Liu, H., Chen, M.-I. and Lu, W.-S. (2015) A Controlled Pilot Trial of Two Commercial Video Games for Rehabilitation of arm Function after Stroke. Clinical Rehabilitation, 29, 674-682. http://dx.doi.org/10.1177/0269215514554115

[10] Laver, K., Ratcliffe, J., George, S., Burgess, L. and Crotty, M. (2011) Is the Nintendo Wii Fit Really Acceptable to Older People? A Discrete Choice Experiment.BMC Geriatrics, 11, 64. http://dx.doi.org/10.1186/1471-2318-11-64

[11] Lewis, G.N. and Rosie, J.A. (2012) Virtual Reality Games for Movement Rehabilitation in Neurological Conditions: How Do We Meet the Needs and Expectations of the Users? Disability \& Rehabilitation, 34, 1880-1886. http://dx.doi.org/10.3109/09638288.2012.670036

[12] Critical Appraisal Skills Programme (CASP) (2013) CASP Qualitative Research Checklist: 10 Questions to Help You Make Sense of Qualitative Research. http://www.casp-uk.net/\#!casp-tools-checklists/c18f8

[13] Tong, A., Flemming, K., McInnes, E., Oliver, S. and Craig, J. (2012) Enhancing Transparency in Reporting the Synthesis of Qualitative Research: ENTREQ. BMC Medical Research Methodology, 12, 181. http://dx.doi.org/10.1186/1471-2288-12-181

\section{Submit or recommend next manuscript to SCIRP and we will provide best service for you:}

Accepting pre-submission inquiries through Email, Facebook, LinkedIn, Twitter, etc.

A wide selection of journals (inclusive of 9 subjects, more than 200 journals)

Providing 24-hour high-quality service

User-friendly online submission system

Fair and swift peer-review system

Efficient typesetting and proofreading procedure

Display of the result of downloads and visits, as well as the number of cited articles

Maximum dissemination of your research work

Submit your manuscript at: http://papersubmission.scirp.org/ 